\title{
The SGLT2 inhibitor dapagliflozin in heart failure with preserved ejection fraction: a multicenter randomized trial
}

\author{
Michael E. Nassifi,2, Sheryl L. Windsor ${ }^{1}$, Barry A. Borlaug ${ }^{(3}{ }^{3}$, Dalane W. Kitzman ${ }^{4}$, Sanjiv J. Shah ${ }^{5}$, \\ Fengming Tang1, Yevgeniy Khariton ${ }^{1,2}$, Ali O. Malik ${ }^{1,2}$, Taiyeb Khumri', Guillermo Umpierrez ${ }^{6}$, \\ Sumant Lamba7, Kavita Sharma ${ }^{8}$, Sadiya S. Khan ${ }^{5}$, Lokesh Chandra ${ }^{9}$, Robert A. Gordon ${ }^{10}$, \\ John J. Ryan"1, Sunit-Preet Chaudhry ${ }^{12}$, Susan M. Joseph ${ }^{13}$, Chen H. Chow ${ }^{14}$, Manreet K. Kanwar ${ }^{15}$, \\ Michael Pursley ${ }^{16}$, Elias S. Siraj ${ }^{17}$, Gregory D. Lewis ${ }^{18}$, Barry S. Clemson ${ }^{19}$, Michael Fong ${ }^{20}$ and \\ Mikhail N. Kosiborod $1,2,21,22 \bowtie$
}

Patients with heart failure and preserved ejection fraction (HFpEF) have a high burden of symptoms and functional limitations, and have a poor quality of life. By targeting cardiometabolic abmormalities, sodium glucose cotransporter 2 (SGLT2) inhibitors may improve these impairments. In this multicenter, randomized trial of patients with HFpEF (NCT03030235), we evaluated whether the SGLT2 inhibitor dapagliflozin improves the primary endpoint of Kansas City Cardiomyopathy Questionnaire Clinical Summary Score (KCCQ-CS), a measure of heart failure-related health status, at 12 weeks after treatment initiation. Secondary endpoints included the 6-minute walk test (6MWT), KCCQ Overall Summary Score (KCCQ-OS), clinically meaningful changes in KCCQ-CS and -OS, and changes in weight, natriuretic peptides, glycated hemoglobin and systolic blood pressure. In total, 324 patients were randomized to dapagliflozin or placebo. Dapagliflozin improved KCCQ-CS (effect size, 5.8 points (95\% con-

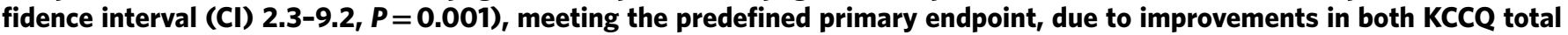
symptom score (KCCQ-TS) $(5.8$ points $(95 \% \mathrm{Cl} 2.0-9.6, P=0.003))$ and physical limitations scores $(5.3$ points $(95 \% \mathrm{Cl} 0.7-$ 10.0, $P=0.026)$ ). Dapagliflozin also improved 6MWT (mean effect size of $20.1 \mathrm{~m}(95 \% \mathrm{Cl} 5.6-34.7, P=0.007)$ ), KCCQ-0S (4.5 points $(95 \% \mathrm{Cl} 1.1-7.8, P=0.009)$ ), proportion of participants with 5-point or greater improvements in KCCQ-OS (odds ratio $(O R)=1.73(95 \% \mathrm{Cl} 1.05-2.85, P=0.03)$ ) and reduced weight (mean effect size, $0.72 \mathrm{~kg}(95 \% \mathrm{Cl} 0.01-1.42, P=0.046)$ ). There were no significant differences in other secondary endpoints. Adverse events were similar between dapagliflozin and placebo (44 (27.2\%) versus $38(23.5 \%)$ patients, respectively). These results indicate that 12 weeks of dapagliflozin treatment significantly improved patient-reported symptoms, physical limitations and exercise function and was well tolerated in chronic HFpEF.

1 eart failure (HF) with preserved ejection fraction (HFpEF) accounts for the majority of all HF in the community, and its prevalence is increasing as the population ages ${ }^{1,2}$. Patients with HFpEF experience an especially high burden of debilitating symptoms and physical limitations ${ }^{3}$. Improving health status (symptoms, functional status and quality of life) is therefore a key goal of HFpEF management, and is increasingly emphasized by practice guidelines and regulators ${ }^{4-7}$. To date, the wide range of pharmacotherapies tested have had minimal impact on these key outcomes, highlighting a critical unmet need.

Impaired health status in HFpEF is strongly linked to cardiometabolic abnormalities ${ }^{8,9}$. SGLT2 inhibitors target cardiometabolic conditions through a variety of mechanisms, and have been shown to reduce the risk of cardiovascular (CV) death or worsening HF, and to improve health status in patients with $\mathrm{HF}$ with reduced ejection fraction (HFrEF), regardless of diabetes status ${ }^{10-13}$. Although initial data from the outcome trials of empagliflozin and sotagliflozin (mixed SGLT1/2 inhibitor) suggest that they also reduce the risk of CV death and HF hospitalization in patients with HFpEF, the effects of SGLT2 inhibitors on patient-reported symptoms, physical limitations and objectively measured exercise function in this patient group remain uncertain ${ }^{14,15}$.

The PRESERVED-HF trial was designed to address this important knowledge gap by testing the hypothesis that treatment with

\footnotetext{
'Saint Luke's Mid America Heart Institute, Kansas City, MO, USA. 'University of Missouri-Kansas City, Kansas City, MO, USA. ${ }^{3}$ Department of Cardiovascular Medicine, Mayo Clinic, Rochester, MN, USA. ${ }^{4}$ Department of Internal Medicine, Sections on Cardiovascular Medicine and Geriatrics, Wake Forest School of Medicine, Winston-Salem, NC, USA. ${ }^{5}$ Division of Cardiology, Department of Medicine and Bluhm Cardiovascular Institute, Northwestern University Feinberg School of Medicine, Chicago, IL, USA. ${ }^{6}$ Emory University, Atlanta, GA, USA. ${ }^{7}$ First Coast Cardiovascular Institute, Jacksonville, FL, USA. ${ }^{8}$ Johns Hopkins University School of Medicine, Baltimore, MD, USA. ${ }^{9}$ Chicago Medical Research, Hazel Crest, IL, USA. ${ }^{10}$ NorthShore University HealthSystem, Evanston, IL, USA. "'University of Utah, Salt Lake City, UT, USA. ${ }^{12}$ Ascension St. Vincent, Indianapolis, IN, USA. ${ }^{13}$ Division of Cardiovascular Medicine, University of Maryland School of Medicine, Baltimore, MD, USA. ${ }^{14}$ Stormont Vail Health, Topeka, KS, USA. ${ }^{15}$ Cardiovascular Institute, Allegheny Health Network, Pittsburgh, PA, USA. ${ }^{16} \mathrm{Heart}$ Group of the Eastern Shore, Fairhope, AL, USA. ${ }^{17}$ Eastern Virginia Medical School, Norfolk, VA, USA. ${ }^{18}$ Cardiology Division, Massachusetts General Hospital, Boston, MA, USA. ${ }^{19}$ OSF HealthCare Cardiovascular Institute, Peoria, IL, USA. ${ }^{20}$ University of Southern California, Los Angeles, CA, USA. ${ }^{21}$ The George Institute for Global Health, Sydney, New South Wales, Australia. ${ }^{22}$ University of New South Wales, Sydney, New South Wales, Australia.凶e-mail: mkosiborod@saint-lukes.org
} 


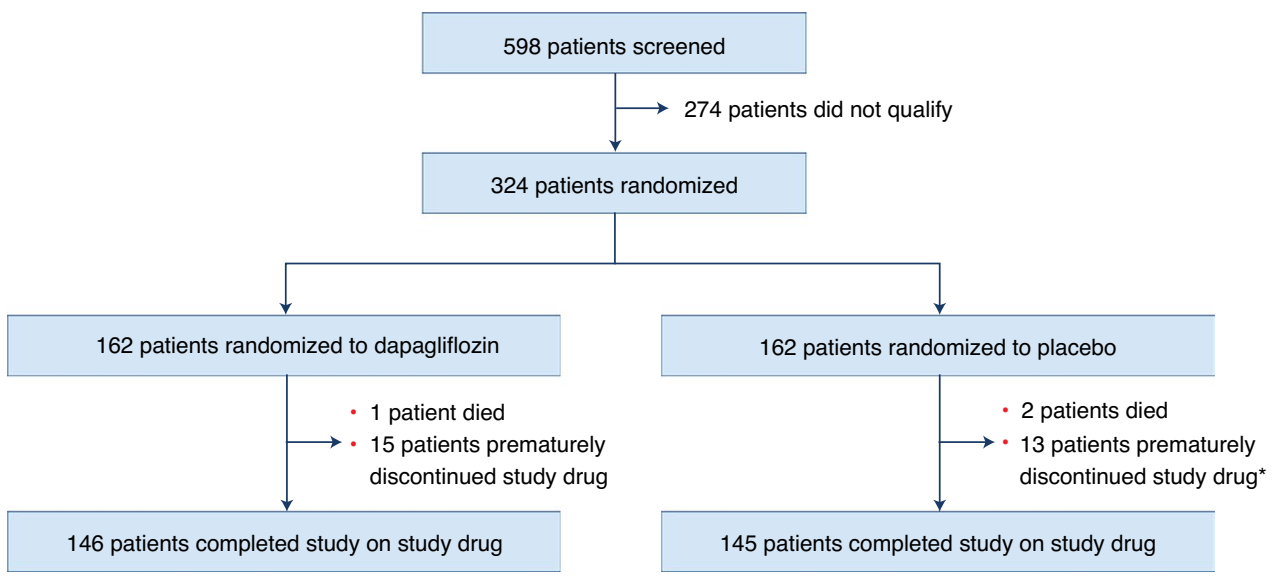

Fig. 1 | Trial flow chart. Breakdown of patients in study. ${ }^{\star}$ For two patients in the placebo group, no data were available on adherence with study medication at 12 weeks.

the SGLT2 inhibitor dapagliflozin will improve symptoms, physical limitations and exercise function in patients with well-phenotyped HFpEF, both with and without type 2 diabetes (T2D).

\section{Results}

Patients. Between March 2017 and May 2021 a total of 598 patients were screened, of which 324 qualified and were randomized: 162 to dapagliflozin and 162 to placebo (Fig. 1). Baseline characteristics were generally well balanced between the two groups (Table 1). Overall, median age was $70.0(63.0,77.0)$ years, $57 \%$ of patients were women and $30 \%$ African American. The median duration of HF was $3.0(1.0,6.5)$ years and $56 \%$ had been hospitalized for HF at least once before study enrollment. Overall, 56\% had T2D and $53 \%$ had AF; median body mass index (BMI) was 34.7 (interquartile range (IQR), 30.1-41.5). New York Heart Association (NYHA) class II symptoms were present in 57\%, with class III/IV symptoms in $42 \%$. Baseline pharmacotherapy included mineralocorticoid antagonists (MRA) in 36\%, angiotensin-converting enzyme inhibitor (ACE-I), angiotensin II receptor blocker (ARB) or angiotensin receptor neprilysin inhibitor (ARNI) in 62\% and loop diuretics in $88 \%$ of patients (with the remainder receiving thiazide diuretics, potassium-sparing diuretics or both). Those randomized to dapagliflozin were more likely to be on loop diuretics (93 versus $83 \%$ ) and less likely to be on MRA (31 versus $42 \%$ ) at baseline. Mean estimated glomerular filtration rate (eGFR) was $55(41,69)$ $\mathrm{ml} \mathrm{min}{ }^{-1} 1.73 \mathrm{~m}^{-2}$, median N-terminal pro B-type natriuretic peptide (NTproBNP) $671.0(\mathrm{IQR}=355.0,1297.0) \mathrm{pg} \mathrm{ml}^{-1}$ and median left ventricular ejection fraction (LVEF) was $60(55,65) \%$.

Premature, permanent treatment discontinuation for reasons other than death occurred in 15 dapagliflozin-treated and 13 placebo-treated patients, resulting in 146 and 145 patients in the dapagliflozin and placebo groups, respectively, completing the trial on study medication (Fig. 1). Safety follow-up was completed in all patients; no patients withdrew consent or were lost to follow-up, and the vital status was known for all participants.

Primary endpoint. The primary endpoint was available in 304 $(93.8 \%)$ patients at 12 weeks $(152(93.8 \%)$ in the dapagliflozin group and $152(93.8 \%)$ in the placebo group). Dapagliflozin improved KCCQ-CS at 12 weeks (effect size, 5.8 points (95\% CI 2.3-9.2), $P=0.001$; Table 2 and Fig. 2a). This was due to improvements in both symptoms (effect size for KCCQ-TS, 5.8 points (95\% CI 2.09.6), $P=0.003$ ) and physical limitations (effect size for KCCQ-PL, 5.3 points (95\% CI $0.7-10.0), P=0.026$; Fig. $2 b, c$, respectively). The results were consistent within subgroups of patients with and with- out T2D, ejection fraction above and below $60 \%$ as well as across all other prespecified subgroups (Fig. 2d; all $P$ values for interaction are nonsignificant).

Secondary endpoints. The 6MWT results were available for 291 $(89.8 \%)$ patients at 12 weeks (148 (91.4\%) in the dapagliflozin group and $143(88.3 \%)$ in the placebo group). Patients treated with dapagliflozin had an improvement in 6MWT distance at 12 weeks (effect size $20.1 \mathrm{~m}$ (95\% CI 5.6-34.7), $P=0.007$; Table 2 and Fig. $3 \mathrm{a})$ that was proportionally large $(8.2 \%)$ given the very low baseline value $(244.4 \mathrm{~m})$

A numerically greater number of patients treated with dapagliflozin versus placebo had a 5-point or greater improvement in KCCQ-CS at 12 weeks (49.4 versus $38.2 \%$; adjusted OR $=1.64(95 \%$ CI $0.98-2.75), P=0.06$; Supplementary Table 1). In the supportive responder analysis, fewer patients treated with dapagliflozin versus placebo experienced deterioration or no change, while a greater proportion of patients treated with dapagliflozin experienced improvement in KCCQ-CS ( $P=0.01$; Fig. 3b).

Results were similar when using KCCQ-OS, with $45.4 \%$ of dapagliflozin-treated patients experiencing 5-point or greater improvement at 12 weeks versus $34.9 \%$ with placebo (adjusted $\mathrm{OR}=1.73,95 \%$ CI 1.05-2.85, $P=0.03$; Supplementary Table 1). Mean KCCQ-OS was also higher with dapagliflozin versus placebo at 12 weeks (adjusted difference, 4.5 points (95\% CI 1.1-7.8) versus placebo, $P=0.009$; Table 2 and Fig. 3 c).

Dapagliflozin also resulted in greater weight loss at 12 weeks (effect size, $0.72 \mathrm{~kg}$ (95\% CI 0.01-1.42), $P=0.046$; Table 2). There were no significant between-group differences in other secondary endpoints, including NTproBNP and BNP; proportion of patients with $20 \%$ or greater decrease in NTproBNP; proportion of patients with both a 5-point or greater increase in KCCQ-CS and $20 \%$ or greater decrease in NTproBNP; hemoglobin A1c (HbAlc); and systolic blood pressure at 12 weeks (Table 2 and Supplementary Table 1).

Exploratory clinical endpoints. In total, nine patients (5.6\%) in each of the treatment groups had adjudicated events of HF hospitalizations or urgent HF visits.

Safety outcomes. The data regarding patients with reportable adverse events are shown in Table 3. One death occurred in the dapagliflozin group and two in the placebo group; all three were adjudicated as non-CV deaths. In the dapagliflozin and placebo groups, respectively, $44(27.2 \%)$ and $38(23.5 \%)$ patients had reported adverse events; 31 (19.1\%) and $22(13.6 \%)$ had serious 
Table 1 | Baseline characteristics

\begin{tabular}{|c|c|c|}
\hline Baseline characteristics & $\begin{array}{l}\text { Dapagliflozin } \\
(n=162)\end{array}$ & Placebo $(n=162)$ \\
\hline \multicolumn{3}{|l|}{ Demographics } \\
\hline Age, years & $69(64,77)$ & $71(63,78)$ \\
\hline Women & $92(56.8 \%)$ & $92(56.8 \%)$ \\
\hline White & $108(67.1 \%)$ & $109(69.0 \%)$ \\
\hline African American & $50(31.1 \%)$ & $47(29.7 \%)$ \\
\hline \multicolumn{3}{|l|}{ Medical history } \\
\hline Duration of $\mathrm{HF}$, years & $3.0(1.1,6.5)$ & $3.2(1.0,6.6)$ \\
\hline Previous hospitalization for $\mathrm{HF}$ & $98(60.5 \%)$ & $83(51.2 \%)$ \\
\hline Ejection fraction, \% & $60(55,65)$ & $60(54,65)$ \\
\hline Ischemic heart disease & $32(19.8 \%)$ & $31(19.1 \%)$ \\
\hline $\mathrm{T} 2 \mathrm{D}$ & $90(55.6 \%)$ & $91(56.2 \%)$ \\
\hline$A F$ & $82(50.6 \%)$ & $89(54.9 \%)$ \\
\hline$I C D$ & $7(4.3 \%)$ & $9(5.6 \%)$ \\
\hline \multicolumn{3}{|l|}{ Baseline HF/CV medications } \\
\hline ACE-I/ARB & $98(60.5 \%)$ & $98(60.5 \%)$ \\
\hline ARNI & $2(1.2 \%)$ & $3(1.9 \%)$ \\
\hline Beta-blockers & $119(73.5 \%)$ & $116(71.6 \%)$ \\
\hline Hydralazine & $25(15.4 \%)$ & $18(11.1 \%)$ \\
\hline Long-acting nitrates & $34(21.0 \%)$ & $27(16.7 \%)$ \\
\hline MRA & $50(30.9 \%)$ & $68(42.0 \%)$ \\
\hline Loop diuretics & $151(93.2 \%)$ & $135(83.3 \%)$ \\
\hline Lipid-lowering agents & $132(81.5 \%)$ & $127(78.4 \%)$ \\
\hline Anticoagulant agents & $71(43.8 \%)$ & $84(51.9 \%)$ \\
\hline \multicolumn{3}{|l|}{ Physical examination } \\
\hline BMI, median IQR & $35.1(30.4,41.8)$ & $34.6(29.7,40.4)$ \\
\hline Heart rate & $70(61,77)$ & $68(62,75)$ \\
\hline Systolic blood pressure & $134(120,152)$ & $132(118,148)$ \\
\hline \multicolumn{3}{|l|}{ Baseline laboratory studies } \\
\hline NTproBNP, pg ml-1 , overall & $641(373,1210)$ & $710(329,1449)$ \\
\hline NTproBNP, pg ml-1, AF & $830(555,1711)$ & $816(481,1687)$ \\
\hline NTproBNP, pg ml-1, no AF & $438(269,750)$ & $485(263,1168)$ \\
\hline BNP, pg ml-1, overall & $137(81,222)$ & $151(90,254)$ \\
\hline $\mathrm{BNP}, \mathrm{pg} \mathrm{ml}^{-1}, \mathrm{AF}$ & $169(109,255)$ & $151(104,258)$ \\
\hline $\mathrm{BNP}, \mathrm{pg} \mathrm{ml^{-1 }}$, no AF & $107(67,179)$ & $161(77,241)$ \\
\hline eGFR, $\mathrm{ml} \mathrm{min}{ }^{-1}$ & $56(42,69)$ & $54(41,69)$ \\
\hline Hemoglobin A1c, \% & $6.0(5.6,7.3)$ & $6.2(5.6,7.1)$ \\
\hline Hemoglobin, $\mathrm{g} \mathrm{dl}^{-1}$ & $12.7(11.5,13.9)$ & $12.6(11.6,13.8)$ \\
\hline \multicolumn{3}{|l|}{ Functional measures } \\
\hline NYHA Class II & $96(59.3 \%)$ & $90(55.6 \%)$ \\
\hline NYHA Class III/IV & $65(40.1 \%)$ & $72(44.4 \%)$ \\
\hline KCCQ-OS & $63.2 \pm 20.4$ & $62.3 \pm 20.6$ \\
\hline KCCQ-CS & $63.4 \pm 19.7$ & $61.8 \pm 20.3$ \\
\hline $6 \mathrm{MWT}(\mathrm{m})$, median $(\mathrm{IQR})$ & $244(165,329)$ & $244(154,317)$ \\
\hline
\end{tabular}

Values are shown as absolute numbers (percentages) and median (IQR) or mean \pm s.d. ICD, internal cardiac defibrillator. *Blood pressure and heart rate were measured from noninvasive cuff measumrents for patients in sinus rhythm and from manual pulse and blood pressure for patients in AF.

adverse events; 18 (11.1\%) and 15 (9.3\%) had adverse events resulting in discontinuation of study medication; and 7 (4.3\%) versus 8 (4.9\%) had drug adverse events (those considered by the
Table 2 | Primary and secondary endpoints at 12 weeks after treatment initiation

\begin{tabular}{|c|c|c|c|c|}
\hline $\begin{array}{l}\text { Continuous } \\
\text { secondary } \\
\text { endpoints }\end{array}$ & $\begin{array}{l}\text { Dapagliflozin } \\
(n=162)\end{array}$ & $\begin{array}{l}\text { Placebo } \\
(n=162)\end{array}$ & $\begin{array}{l}\text { Effect } \\
\text { size }\end{array}$ & $P$ value \\
\hline KCCQ-CS, mean ${ }^{\mathrm{a}}$ & $\begin{array}{l}68.6(66.2, \\
71.0)\end{array}$ & $\begin{array}{l}62.8(60.4 \\
65.3)\end{array}$ & $\begin{array}{l}5.8(2.3 \\
9.2)\end{array}$ & 0.001 \\
\hline KCCQ-OS, mean ${ }^{\mathrm{a}}$ & $68.9(66.5,71.3)$ & $\begin{array}{l}64.5(62.1 \\
66.8)\end{array}$ & $\begin{array}{l}4.5(1.1 \\
7.8)\end{array}$ & 0.009 \\
\hline $6 \mathrm{MWT}$, mean, $\mathrm{m}^{\mathrm{a}}$ & $262(252,272)$ & $\begin{array}{l}242(232, \\
252)\end{array}$ & $\begin{array}{l}20.1 \\
(5.6 \\
34.7)\end{array}$ & 0.007 \\
\hline $\begin{array}{l}\text { NTproBNP, mean, } \\
\text { pg ml-1a }\end{array}$ & $733(673,799)$ & $\begin{array}{l}739(678, \\
805)\end{array}$ & $\begin{array}{l}0.99 \\
(0.88 \\
1.12)^{b}\end{array}$ & 0.900 \\
\hline $\mathrm{BNP}$, mean, $\mathrm{pg} \mathrm{ml}^{-1 \mathrm{a}}$ & $147(136,160)$ & $\begin{array}{l}147(136, \\
160)\end{array}$ & $\begin{array}{l}1.00 \\
(0.89 \\
1.12)^{b}\end{array}$ & 0.990 \\
\hline $\begin{array}{l}\text { Systolic blood } \\
\text { pressure, mean, } \\
\mathrm{mmHg}^{\mathrm{a}}\end{array}$ & $133(130,135)$ & $\begin{array}{l}133(131, \\
136)\end{array}$ & $\begin{array}{l}-0.6 \\
(-4.4 \\
3.3)\end{array}$ & 0.780 \\
\hline Weight, mean, kga & $\begin{array}{l}101.3(100.9 \\
101.8)\end{array}$ & $\begin{array}{l}102.1(101.6, \\
102.6)\end{array}$ & $\begin{array}{l}-0.72 \\
(-1.42 \\
-0.01)\end{array}$ & 0.046 \\
\hline
\end{tabular}

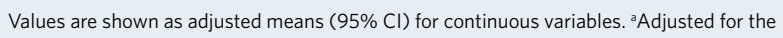
corresponding baseline value, history of T2D, sex, AF, baseline eGFR and LVEF. ${ }^{\text {bRatio of }}$ dapagliflozin compared to placebo.

investigator to be due to, and resulting in, permanent discontinuation of the investigational product). Adverse events of volume depletion were reported in $11(6.8 \%)$ versus $7(4.3 \%)$ patients; and acute kidney injury in $5(3.1 \%)$ versus $5(3.1 \%)$ in the dapagliflozin and placebo groups, respectively. No events of diabetic ketoacidosis (DKA), severe hypoglycemia or lower limb amputation occurred during the trial.

\section{Discussion}

In this multicenter, double-blind, randomized, placebo-controlled trial in patients with HFpEF, treatment with dapagliflozin improved HF-related symptoms and physical limitations as measured by KCCQ-CS after only 12 weeks of treatment. The magnitude of these benefits was clinically meaningful, statistically significant and consistent across all prespecified subgroups, including patients with and without T2D and those with ejection fraction above and below $60 \%$. Patients treated with dapagliflozin also had a significant, clinically meaningful 20-m improvement in 6MWT distance. To our knowledge this may represent the first clinical trial to show a significant benefit of SGLT2 inhibitors on both patient-reported symptoms and physical limitations, as well as objectively measured physical function, in individuals with HFpEF.

Improvement in symptoms and physical function in HFpEF are key goals of management, given that this population has especially poor health status ${ }^{16}$. Although SGLT2 inhibitors have been shown to improve symptoms in individuals with $\mathrm{HFrEF}^{10-12}$, their effects on health status in symptomatic individuals with chronic HFpEF are not established ${ }^{15,17}$. The magnitude of benefit (5.8- and 4.5-point improvement in KCCQ-CS and KCCQ-OS, respectively) with dapagliflozin is especially notable, as previous therapies tested in HFpEF have generally not produced a clinically meaningful improvement in health status. Specifically, in the TOPCAT trial, treatment with spironolactone resulted in a 1.5-point improvement in KCCQ-OS versus placebo and, in the PARAGON trial, treatment with sacubitril valsartan resulted in a 1-point higher KCCQ-OS compared 


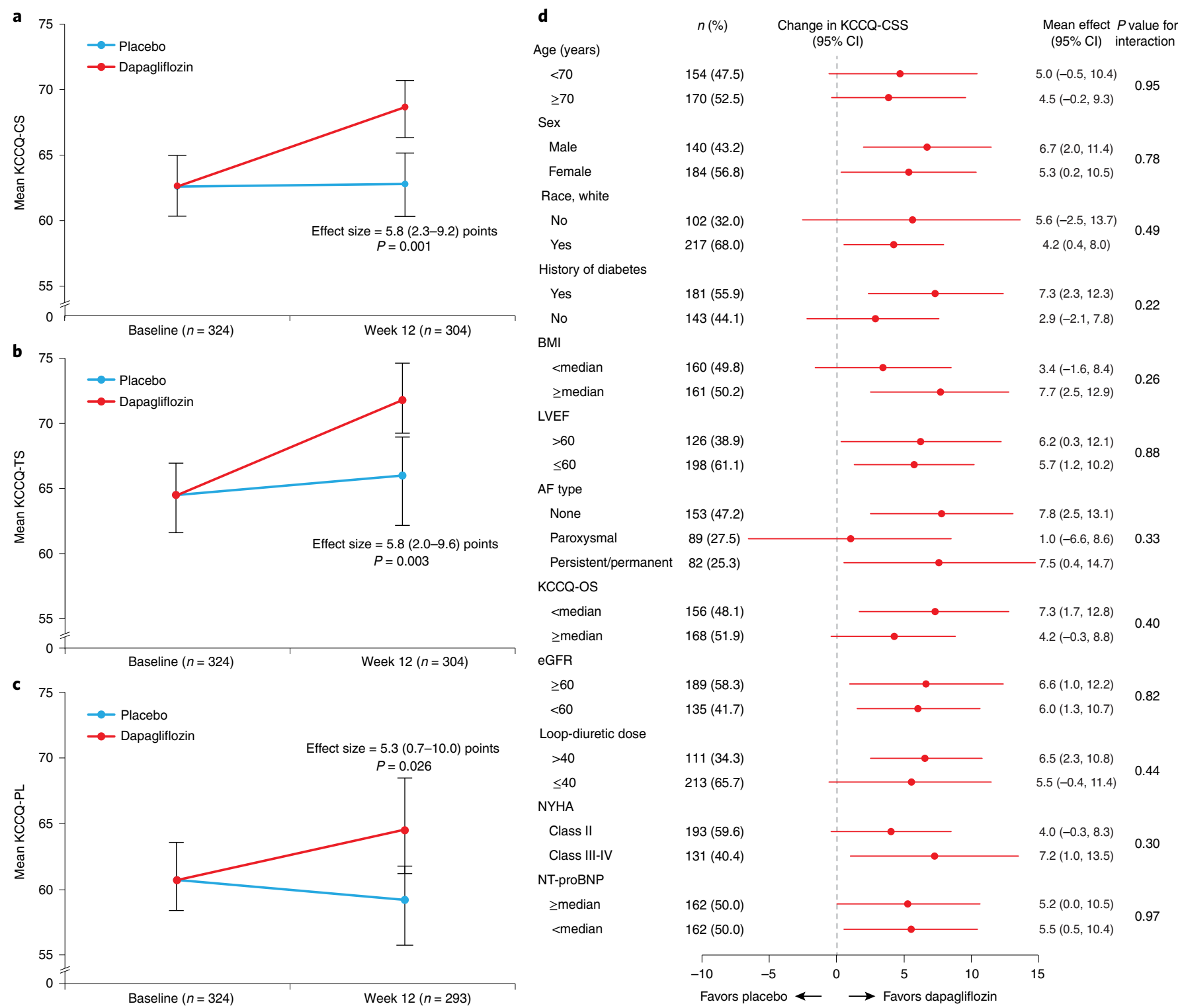

Fig. 2 | Effects of dapagliflozin on the primary endpoint and its components. a-d, Effects of dapagliflozin on the primary endpoint and its components. Effects of dapagliflozin versus placebo at 12 weeks on KCCQ-CS (a), KCCQ-TS (b), KCCQ-physical limitations score (KCCQ-PL) (c) and KCCQ-CS by subgroup (d). Units for loop diuretic dose (d), mg furosemide equivalents. Data are presented as mean values with $95 \% \mathrm{Cl}$. a-c, An $\mathrm{F}$-test was used in the data analysis. All $P$ values are two-sided, with no adjustments made for multiple comparisons.

with valsartan ${ }^{18,19}$. Our results are further buttressed by the findings in responder analyses using KCCQ-CS, which indicated that dapagliflozin-treated patients were more likely to experience a very large (20-point or greater) improvement in health status and less likely to have worsening health status.

These results were consistent within subgroups of patients with and without T2D, as well as across all other prespecified subgroups. Of note, our study population was diverse with $57 \%$ women and $30 \%$ African American participants-consistent with the population-level demographic characteristics of individuals with HFpEF in the United States ${ }^{1,2}$. Notably, the health status benefits of dapagliflozin were consistent in each of these important subgroups that have traditionally been under-represented in HF trials and that have a great need for efficacious therapies. The use of baseline medical therapies observed in PRESERVED-HF was overall similar to that seen in several large, contemporary HFpEF trials. Specifically, $>99 \%$ of participants in PRESERVED-HF were receiving diuretics (versus 86 and $96 \%$ in EMPEROR-PRESERVED and PARAGON-HF, respectively) and $36 \%$ of participants in PRESERVED-HF were receiving mineralocorticoid receptor antagonists, a rate that was greater than in PARAGON-HF (24\%) and similar to that in EMPEROR-PRESERVED (37\%) $)^{15,19,20}$.

Our results differ from one previous trial of similar size and treatment duration ${ }^{21}$. The EMPERIAL-PRESERVED trial evaluated the effects of the SGLT2 inhibitor empagliflozin on symptoms and functional status in HFpEF and reported a nonsignificant, 2.0-point increase in KCCQ-TS with empagliflozin versus placebo, in contrast to the larger (and highly statistically significant) 5.8-point improvement in both KCCQ-TS and KCCQ-CS in the present study ${ }^{21}$. EMPERIAL-PRESERVED also reported a nonsignificant 4-m improvement in 6MWT distance with empagliflozin versus placebo, while we observed a much larger (and statistically significant) 20-m improvement with dapagliflozin versus placebo. The 5.8-point improvement in KCCQ-CS that was observed in PRESERVED-HF 


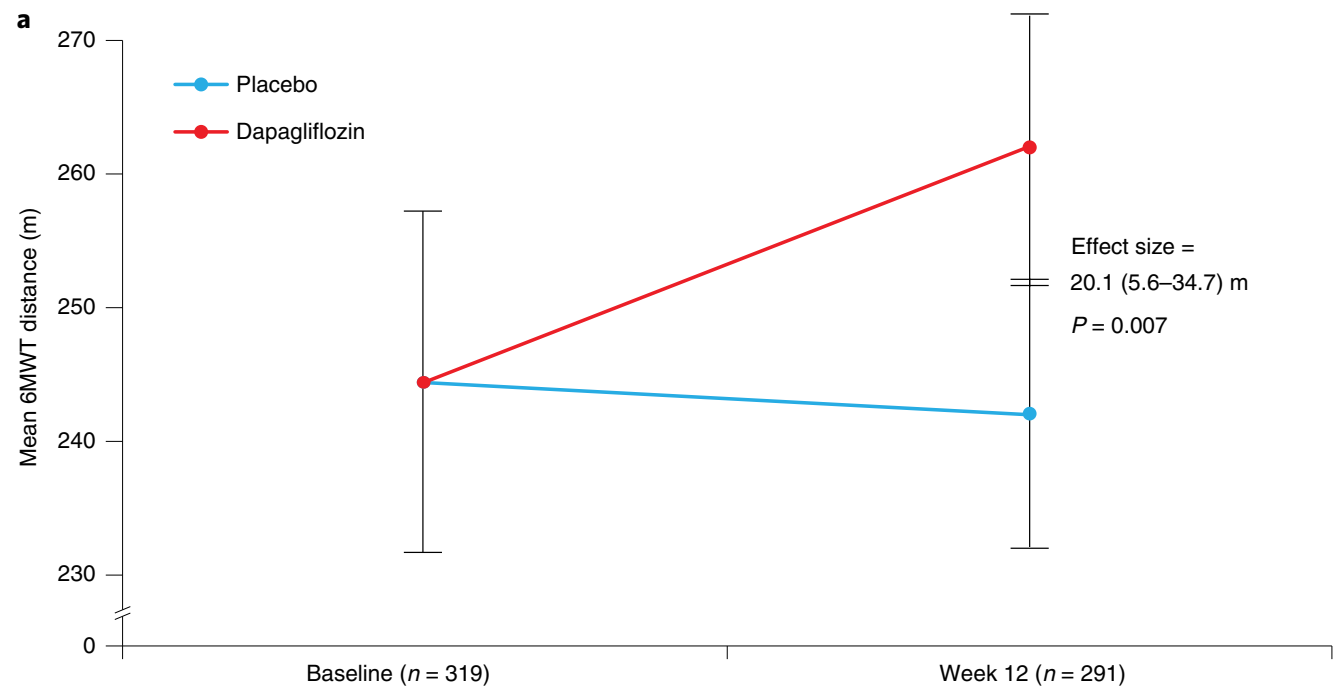

b
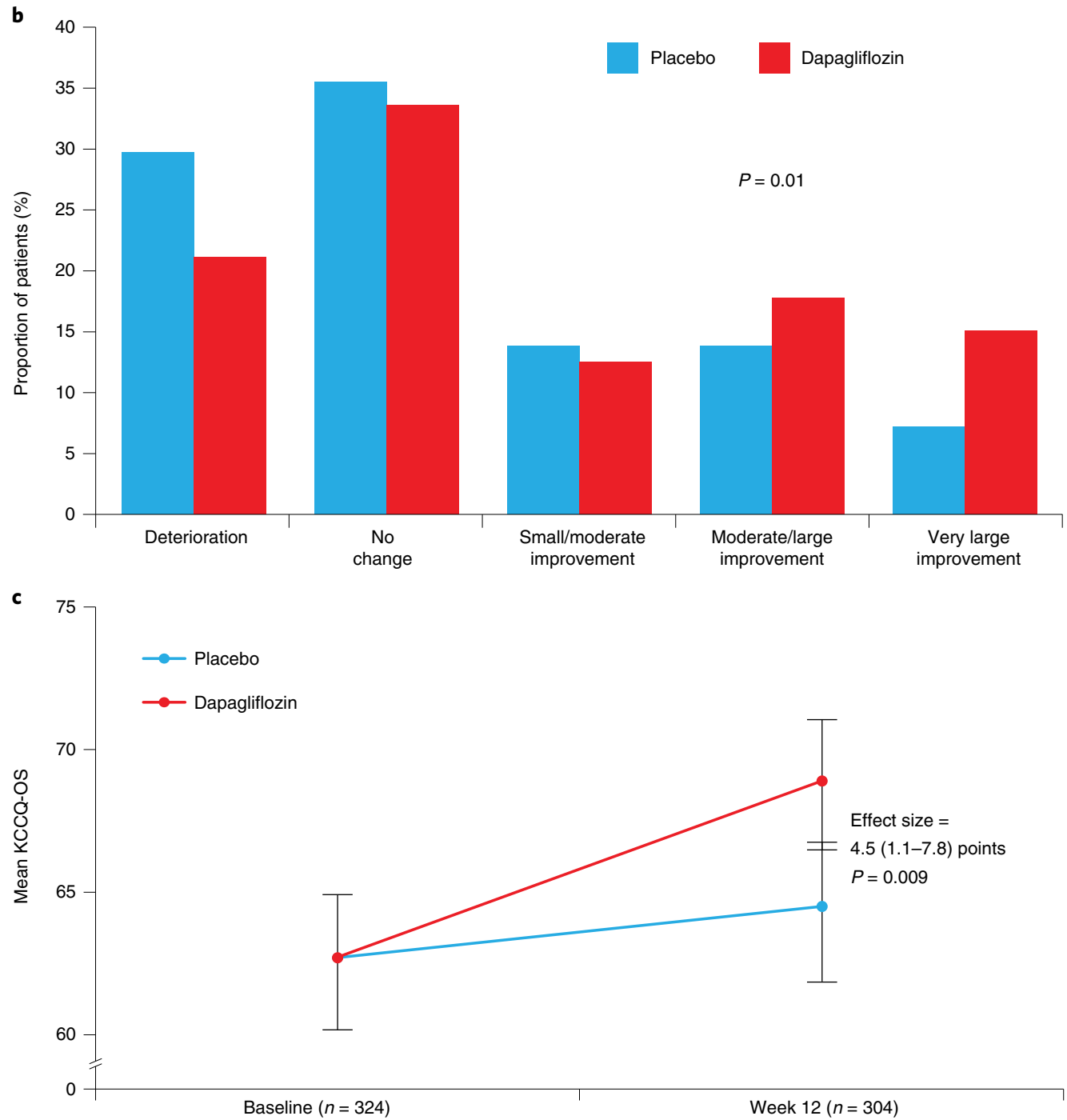

Fig. 3 | Effects of dapagliflozin on selected secondary endpoints and in supportive responder analysis. a-c, Effects of dapagliflozin on selected secondary endpoints and in supportive responder analysis. Effects of dapagliflozin versus placebo at 12 weeks on 6MWT distance (a), KCCQ-CS responder analysis (b) and KCCQ-OS (c). Data are presented as mean values with $95 \% \mathrm{Cl}$. a,c, An F-test was used in data analysis; b, a chi-square test was used in data analysis All $P$ values are two-sided, with no adjustments made for multiple comparisons.

is also larger than the 1.3-point difference in KCCQ-CS seen with empagliflozin versus placebo in EMPEROR-PRESERVED, a large global outcome trial that demonstrated a significant reduction in the primary endpoint of $\mathrm{CV}$ death or hospitalizations for $\mathrm{HF}$ in participants with chronic HFpEF ${ }^{15}$. However, it should be noted that KCCQ-CS was assessed at 1 year in EMPEROR-PRESERVED 
versus at 12 weeks in both EMPERIAL-PRESERVED and PRESERVED-HF.

One potential explanation for these discrepancies between EMPERIAL-PRESERVED, EMPEROR-PRESERVED and PRESERVED-HF may be differences in baseline characteristics of the study participants. Specifically, PRESERVED-HF (versus EMPERIAL-PRESERVED and EMPEROR-PRESERVED) included a higher proportion of participants who were women (58 versus 43 and $45 \%$, respectively) and African American (30 versus 10 and 4\%), with notably higher BMI (34.7 versus 29.6 and $29.8 \mathrm{~kg} \mathrm{~m}^{-2}$ ), all characteristics that more closely match those of patients with HFpEF in the US population ${ }^{1}$. Participants in PRESERVED-HF (versus those in EMPERIAL-PRESERVED and EMPEROR-PRESERVED) also had a considerably greater degree of symptomatic and functional impairment as measured by NYHA class ( 42 versus 22 and $20 \%$ with NYHA class III/IV, respectively), and by $6 \mathrm{MWT}$ distance (244 versus $298 \mathrm{~m}$ in EMPERIAL-PRESERVED but not assessed in EMPEROR-PRESERVED), characteristics that have been strongly linked to poorer quality of life ${ }^{8}$. PRESERVED-HF (as compared with EMPERIAL-PRESERVED, but not with EMPEROR-PRESERVED) also included a higher propotion of participants who required loop-diuretic therapy (88 versus 72 and $86 \%$, respectively) and a higher proportion of participants with AF (53 versus 30 and $52 \%$ ), which is associated with poorer cardiac reserve and more right-sided HF and pulmonary hypertension ${ }^{22}$. Importantly, our trial was also conducted exclusively at sites in the United States whereas EMPERIAL-PRESERVED enrolled patients from Western and Eastern Europe, Australia and Canada, as well as from the United States, and EMPEROR-PRESERVED had global participation. While it is possible that there are pharmacodynamic differences between empagliflozin and dapagliflozin, this explanation seems less likely as both agents have been found to provide similar benefits on the composite of CV death or hospitalization for HF in large outcome trials of individuals with $\mathrm{HFrEF}^{11,12}$.

The observed improvement in 6MWT is relatively unique and highly clinically relevant. Even when patients with HFpEF are stable and well compensated, they have a markedly impaired objectively measured physical function ${ }^{23}$. Impaired physical function is an independent predictor of poorer quality of life, hospitalizations, loss of independence, nursing home placement and death. Formal patient interviews indicate that patients with HF value improved physical function at least equally with avoidance of death ${ }^{24,25}$. To date, 12 trials have formally tested a variety of medications in $\mathrm{HFpEF}$ for exercise function outcomes, as measured by 6MWT or cardiopulmonary exercise testing, and nearly all have been neutral $^{23}$. Furthermore, all five classes of agents proven to improve clinical events in HFrEF have minimal impact on exercise function. The magnitude of the increase in 6MWT distance that we observed is proportionally large $(8.2 \%)$ and is greater than that observed in the HF-ACTION trial of exercise training in HFrEF, where it was associated with an improvement in clinical events, and is similar in magnitude to that observed in exercise training trials of $\mathrm{HFpEF}^{26,27}$.

Several potential mechanisms may explain the clinical benefits of dapagliflozin we observed in this trial. First, SGLT2 inhibitors have been shown to rapidly lower pulmonary artery pressure, which aids decongestion and can translate to improvements in both symptoms and exercise function ${ }^{28,29}$. Second, SGLT2 inhibitors may increase myocardial energy production; alleviate systemic microvascular dysfunction, which is prevalent in both the myocardium and skeletal muscle in HFpEF; improve systemic endothelial function; reduce systemic inflammation and oxidative stress; and improve insulin sensitivity and activate fatty acid oxidation in the skeletal muscle ${ }^{30-33}$. Finally, SGLT2 inhibitors also result in modest weight loss. This is of relevance given the high prevalence of overweight/ obesity among patients with HFpEF (>80\%) in the United States,
Table 3 | Safety analyses

\begin{tabular}{|c|c|c|}
\hline & $\begin{array}{l}\text { Dapagliflozin } \\
(n=162)\end{array}$ & $\begin{array}{l}\text { Placebo } \\
(n=162)\end{array}$ \\
\hline All reported adverse events & $44(27.2 \%)$ & $38(23.5 \%)$ \\
\hline Serious adverse events & $31(19.1 \%)$ & $22(13.6 \%)$ \\
\hline $\begin{array}{l}\text { Adverse events resulting } \\
\text { in discontinuation of study } \\
\text { medication }\end{array}$ & $18(11.1 \%)$ & $15(9.3 \%)$ \\
\hline Drug adverse events & $7(4.3 \%)$ & $8(4.9 \%)$ \\
\hline All-cause death & $1(0.6 \%)$ & $2(1.2 \%)$ \\
\hline Nonfatal MI & $0(0 \%)$ & $1(0.6 \%)$ \\
\hline Stroke & $0(0 \%)$ & $1(0.6 \%)$ \\
\hline Acute kidney injury & $5(3.1 \%)$ & $5(3.1 \%)$ \\
\hline DKA & $0(0 \%)$ & $0(0 \%)$ \\
\hline Volume depletion events & $11(6.8 \%)$ & $7(4.3 \%)$ \\
\hline Severe hypoglycemic events & $0(0 \%)$ & $0(0 \%)$ \\
\hline Lower limb amputations & $0(0 \%)$ & $0(0 \%)$ \\
\hline
\end{tabular}

Values are shown as absolute numbers (percentages) for patients with events.

which was clearly reflected in our trial with an average baseline BMI of 35 (ref. ${ }^{34}$ ).

Consistent with previous data from comparably sized trials, dapagliflozin treatment had no significant effect on natriuretic peptides $^{10}$. Although natriuretic peptide levels are predictive of prognosis in HFpEF, they are known to be considerably lower than in HFrEF and previous studies have shown little relationship between natriuretic peptides and health status ${ }^{8,35}$. Future HFpEF trials may benefit from focusing on patient-centered outcomes such as health status and exercise function that are more relevant to a patient's journey, as was done in PRESERVED-HF. Dapagliflozin's tolerability profile was also generally consistent with previous SGLT2 inhibitor trials, with no new safety signals identified. Reassuringly, we did not observe any events of DKA or severe hypoglycemia (although about half of the patients did not have T2D and the duration of the study was short).

Despite the strengths in study design and execution, our findings should be interpreted in the context of several potential limitations. First, its relatively short duration of follow-up precludes assessment regarding the durability of the observed benefit on HF-disease-specific symptoms or functional status. Second, all patients were enrolled at sites in the United States and, while this makes the study applicable to the US population with HFpEF, its generalizability outside that country is uncertain. Additional data from global trials of SGLT2 inhibitors, including the recently completed EMPEROR-PRESERVED and the ongoing DELIVER trial, will ultimately address some of these limitations. Nevertheless, even the short-term improvements in health status and exercise function shown here with dapagliflozin represent an important therapeutic advance given the lack of available effective treatments for HFpEF.

In conclusion, dapagliflozin significantly improved symptoms, physical limitations and objectively measured exercise function in patients with HFpEF. The magnitude of treatment benefits was large, clinically meaningful and statistically significant, and consistent across all prespecified subgroups.

\section{Online content}

Any methods, additional references, Nature Research reporting summaries, source data, extended data, supplementary information, acknowledgements, peer review information; details of author contributions and competing interests; and statements of 
data and code availability are available at https://doi.org/10.1038/ s41591-021-01536-x.

Received: 19 August 2021; Accepted: 10 September 2021; Published online: 28 October 2021

\section{References}

1. Dunlay, S. M., Roger, V. L. \& Redfield, M. M. Epidemiology of heart failure with preserved ejection fraction. Nat. Rev. Cardiol. 14, 591-602 (2017).

2. Pfeffer, M. A., Shah, A. M. \& Borlaug, B. A. Heart failure with preserved ejection fraction in perspective. Circ. Res. 124, 1598-1617 (2019).

3. Warraich, H. J. et al. Physical function, frailty, cognition, depression, and quality of life in hospitalized adults $\geq 60$ years with acute decompensated heart failure with preserved versus reduced ejection fraction. Circ. Heart Fail. 11, e005254 (2018).

4. Reddy, P. \& Dunn, A. B. The effect of beta-blockers on health-related quality of life in patients with heart failure. Pharmacotherapy 20, 679-689 (2000).

5. Tsevat, J. et al. Using health-related quality-of-life information: clinical encounters, clinical trials, and health policy. J. Gen. Intern. Med. 9, 576-582 (1994)

6. Lewis, E. F. et al. Preferences for quality of life or survival expressed by patients with heart failure. J. Heart Lung Transplant. 20, 1016-1024 (2001).

7. US Food \& Drug Administration. Treatment for heart failure: endpoints for drug development guidance for industry. https://www.fda.gov/ regulatory-information/search-fda-guidance-documents/ treatment-heart-failure-endpoints-drug-development-guidance-industry (2019).

8. Reddy, Y. N. V. et al. Quality of life in heart failure with preserved ejection fraction: importance of obesity, functional capacity, and physical inactivity. Eur. J. Heart Fail. 22, 1009-1018 (2020).

9. Dunlay, S. M. et al. Type 2 diabetes mellitus and heart failure: a scientific statement from the American Heart Association and the Heart Failure Society of America: this statement does not represent an update of the 2017 ACC/ AHA/HFSA heart failure guideline update. Circulation 140, e294-e324 (2019).

10. Nassif, M. E. et al. Dapagliflozin effects on biomarkers, symptoms, and functional status in patients with heart failure with reduced ejection fraction: the DEFINE-HF Trial. Circulation 140, 1463-1476 (2019).

11. McMurray, J. J. V. et al. Dapagliflozin in patients with heart failure and reduced ejection fraction. N. Engl. J. Med. 381, 1995-2008 (2019).

12. Packer, M. et al. EMPEROR-Reduced Trial Investigators. Cardiovascular and renal outcomes with empagliflozin in heart failure. N. Engl. J. Med. 383, 1413-1424 (2020).

13. Cowie, M. R. \& Fisher, M. SGLT2 inhibitors: mechanisms of cardiovascular benefit beyond glycaemic control. Nat. Rev. Cardiol. 17, 761-772 (2020).

14. Bhatt, D. L. et al. Sotagliflozin in patients with diabetes and recent worsening heart failure. N. Engl. J. Med. 384, 117-128 (2020).

15. Anker, S. D. et al. Empagliflozin in heart failure with a preserved ejection fraction. N. Engl. J. Med. https://www.nejm.org/doi/full/10.1056/ NEJMoa2107038 (2021).

16. Chandra, A. et al. Health-related quality of life in heart failure with preserved ejection fraction: the PARAGON-HF trial. JACC Heart Fail. 7, 862-874 (2019).

17. Solomon, S. D. et al. Dapagliflozin in heart failure with preserved and mildly reduced ejection fraction: rationale and design of the DELIVER trial. Eur. J. Heart Fail. 23, 1217-1225 (2021).

18. Lewis, E. F. et al. Impact of spironolactone on longitudinal changes in health-related quality of life in the treatment of preserved cardiac function heart failure with an aldosterone antagonist trial. Circ. Heart Fail. 9, e001937 (2016).

19. Solomon, S. D. et al. Angiotensin-neprilysin inhibition in heart failure with preserved ejection fraction. N. Engl. J. Med. 381, 1609-1620 (2019).
20. Anker, S. D. et al. Baseline characteristics of patients with heart failure with preserved ejection fraction in the EMPEROR-Preserved trial. Eur. J. Heart Fail. 22, 2383-2392 (2020).

21. Abraham, W. T. et al. Effect of empagliflozin on exercise ability and symptoms in heart failure patients with reduced and preserved ejection fraction, with and without type 2 diabetes. Eur. Heart J. 42, 700-710 (2021).

22. Reddy, Y. N. V., Obokata, M., Verbrugge, F. H., Lin, G. \& Borlaug, B. A. Atrial dysfunction in patients with heart failure with preserved ejection fraction and atrial fibrillation. J. Am. Coll. Cardiol. 76, 1051-1064 (2020).

23. Pandey, A. Exercise intolerance and heart failure with preserved ejection fraction (HFpEF) in older adults: JACC state-of-the-art review. J. Am. Coll. Cardiol. 78, 1166-1187 (2021).

24. Reed, S. D. et al. Patients' willingness to accept mitral valve procedure-associated risks varies across severity of heart failure symptoms. Circ. Cardiovasc. Interv. 12, e008051 (2019).

25. Forman, D. E. et al. Prioritizing functional capacity as a principal end point for therapies oriented to older adults with cardiovascular disease: a scientific statement for healthcare professionals from the American Heart Association. Circulation 135, e894-e918 (2017).

26. O'Connor, C. M. et al. Efficacy and safety of exercise training in patients with chronic heart failure: HF-ACTION randomized controlled trial. J. Am. Med. Assoc. 301, 1439-1450 (2009).

27. Pandey, A. et al. Exercise training in patients with heart failure and preserved ejection fraction: meta-analysis of randomized control trials. Circ. Heart Fail. 8, 33-40 (2015).

28. Nassif, M. E. et al. Empagliflozin effects on pulmonary artery pressure in patients with heart failure. Circulation 143, 1673-1686 (2021).

29. Wolsk, E. et al. Resting and exercise haemodynamics in relation to six-minute walk test in patients with heart failure and preserved ejection fraction. Eur. J. Heart Fail. 20, 715-722 (2018).

30. Verma, S. et al. Empagliflozin increases cardiac energy production in diabetes: novel translational insights into the heart failure benefits of SGLT2 inhibitors. JACC Basic Transl. Sci. 3, 575-587 (2018).

31. Juni, R. P. et al. Cardiac microvascular endothelial enhancement of cardiomyocyte function is impaired by inflammation and restored by empagliflozin. JACC Basic Transl. Sci. 4, 575-591 (2019).

32. Shah, S. J. et al. Prevalence and correlates of coronary microvascular dysfunction in heart failure with preserved ejection fraction: PROMIS-HFpEF. Eur. Heart J. 39, 3439-3450 (2018).

33. Nambu, H. et al. Empagliflozin restores lowered exercise endurance capacity via the activation of skeletal muscle fatty acid oxidation in a murine model of heart failure. Eur. J. Pharmacol. 866, 172810 (2020).

34. Obokata, M., Reddy, Y. N. V., Pislaru, S. V., Melenovsky, V. \& Borlaug, B. A. Evidence supporting the existence of a distinct obese phenotype of heart failure with preserved ejection fraction. Circulation 136, 6-19 (2017).

35. van Veldhuisen, D. J. et al. B-type natriuretic peptide and prognosis in heart failure patients with preserved and reduced ejection fraction. J. Am. Coll. Cardiol. 61, 1498-1506 (2013).

Publisher's note Springer Nature remains neutral with regard to jurisdictional claims in published maps and institutional affiliations.

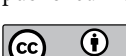

Open Access This article is licensed under a Creative Commons Attribution 4.0 International License, which permits use, sharing, adaptation, distribution and reproduction in any medium or format, as long as you give appropriate credit to the original author(s) and the source, provide a link to the Creative Commons license, and indicate if changes were made. The images or other third party material in this article are included in the article's Creative Commons license, unless indicated otherwise in a credit line to the material. If material is not included in the article's Creative Commons license and your intended use is not permitted by statutory regulation or exceeds the permitted use, you will need to obtain permission directly from the copyright holder. To view a copy of this license, visit http://creativecommons. org/licenses/by/4.0/.

(C) The Author(s) 2021 


\section{Methods}

PRESERVED-HF was a randomized, double-blind, placebo-controlled, multicenter trial of individuals with chronic, symptomatic HFpEF (Supplementary Table 2). The trial was conducted across 26 sites in the United States. The list of participating sites and investigators is given in Supplementary Table 3 and Extended Data Fig. 1. The primary outcome was HF-disease-specific health status as assessed by KCCQ-CS ${ }^{36}$. The study protocol is provided in the Supplementary Data.

PRESERVED-HF was an investigator-initiated trial with the concept developed and the trial sponsored and executed by, the national coordinating center at Saint Luke's Mid America Heart Institute in collaboration with the Executive Committee (Supplementary Table 4), independent of the funding source (AstraZeneca). The study was monitored by an independent data safety and monitoring committee. Institutional review boards approved the study for all sites (Supplementary Table 3 and Extended Data Fig. 1), and all patients provided informed consent for research participation. The trial was conducted in accordance with the ICH E6(R1) Guidelines of Good Clinical Practice and the Declaration of Helsinki.

Patient selection. Full inclusion and exclusion criteria can be found in Supplementary Table 5. Adult ambulatory patients with or without T2D, clinical diagnosis of HFpEF, LVEF $\geq 45 \%$ and NYHA class II-IV symptoms were screened for participation. Patients additionally had to have elevated natriuretic peptides (NTproBNP $\geq 225$ or BNP $\geq 75 \mathrm{pg} \mathrm{ml}^{-1}$; if AF, NTproBNP $\geq 375 \mathrm{pg} \mathrm{ml}^{-1}$ or $\mathrm{BNP} \geq 100 \mathrm{pg} \mathrm{ml}^{-1}$ ); requirement for diuretic therapy (loop, thiazide or potassium-sparing diuretics) and either HF hospitalization or urgent HF visit with intravenous diuretic treatment in the past 12 months; documented elevated filling pressures on right or left heart catheterization; or echocardiographic evidence of structural heart abnormalities. Key exclusion criteria were recent hospitalization (within 7 days) for decompensated HF, eGFR $<20 \mathrm{ml} \mathrm{min}^{-1} 1.73 \mathrm{~m}^{-2}$ at the screening visit, type 1 diabetes or previous history of DKA.

Trial design. Patients considered potentially eligible and who agreed to participate and provided informed consent entered a 2 -week screening phase during which their eligibility was confirmed based on central core laboratory evaluation (NTproBNP $\geq 225 \mathrm{pg} \mathrm{ml}^{-1}$ or BNP $\geq 75 \mathrm{pg} \mathrm{ml}^{-1}$; higher if AF), eGFR $\geq 20 \mathrm{ml} \mathrm{min}^{-1}$ $1.73 \mathrm{~m}^{-2}$ and clinical stability was ensured. Patients confirmed as eligible were randomized in a double-blind fashion-1:1 to oral dapagliflozin $10 \mathrm{mg}$ or matching placebo once daily (Extended Data Fig. 2). Before administration of the first dose of dapagliflozin or placebo, patients underwent a physical examination, trial-related laboratory assessments, completion of the KCCQ and a 6MWT. Patients then entered a 12-week treatment period during which they were followed via four telephone call visits as well as two in-person study visits (at 6 and 12 weeks), at which times repeat physical examination, laboratory assessment (at 6 and 12 weeks), KCCQ and $6 \mathrm{MWT}$ were completed (at 12 weeks). At week 12 , study medication was discontinued and patients were followed for one additional week, at the end of which another in-person study visit was conducted to assess for any intercurrent safety events, and to collect additional laboratory samples.

Outcomes. The primary outcome was KCCQ-CS at 12 weeks. KCCQ-CS was originally a key secondary endpoint, with the original primary outcome defined as mean change in NTproBNP from baseline to 6 and 12 weeks. However, during the trial, compelling external scientific information from a related trial of dapagliflozin in HFrEF showed a greater benefit on patient-reported symptoms and physical limitations (measured using the KCCQ) - outcomes that are more meaningful to patients and clinicians than change in NTproBNP ${ }^{10}$. Accordingly, in March 2020 (17 months before database lock) the protocol was amended to elevate the KCCQ-CS at 12 weeks as the primary endpoint. This decision was made solely by the excutive committee, which was fully blinded at the time.

The KCCQ is a standardized, 23-item, self-administered instrument that quantifies HF-related symptoms (frequency, severity and recent change), physical function, quality of life and social function. KCCQ-CS includes symptoms and physical function (domains considered most likely to be modified by SGLT2 inhibitors). For each domain, the validity, reproducibility, responsiveness and interpretability have been independently established. Scores are transformed to a range of $0-100$, in which higher scores reflect better health status ${ }^{36}$.

The key secondary outcome was the 6MWT, which was conducted according to well-established methods ${ }^{37}$. Other secondary outcomes included the proportion of patients with meaningful (5-point or greater) change in KCCQ-CS and -OS; 6MWT distance; NTproBNP and BNP; proportion of patients with $20 \%$ or greater decrease in NTproBNP and BNP; proportion of patients with both 5-point or greater increase in KCCQ-CS and 20\% or greater decrease in NTproBNP; HbAlc; and weight and systolic blood pressure at 12 weeks. Levels of NTproBNP and BNP, and all other study laboratory assessments, were analyzed at a Quest Diagnostics central laboratory, blinded to treatment assignment (NTproBNP assay Roche electrochemiluminescent method on Elecsys platform, ProBNP II reagent by Roche/Cobas; BNP assay chemiluminescent method on Siemens ADVIA Centaur platform). Exploratory endpoints included the number of hospitalizations for HF or urgent HF visits (Supplementary Table 2).

All serious adverse events were reported by investigators. An independent, blinded clinical events committee adjudicated all deaths, hospitalizations for
HF, urgent HF visits, myocardial infraction (MI) and transient ischemic attack/ stroke events. In addition, investigator-reported adverse events of special interest included acute kidney injury (defined as doubling of serum creatinine), DKA, volume depletion, severe hypoglycemia (defined as blood glucose $<54 \mathrm{mg} \mathrm{dl}^{-1}$ $\left(<3.0 \mathrm{mmoll}^{-1}\right)$ and requiring assistance) and lower limb amputations.

Concomitant medications. The trial was designed to enroll patients receiving standard-of-care therapy for HFpEF. In patients with T2D, plans for reducing the risk of hypoglycemia included a suggested $20 \%$ reduction in the total daily dose of insulin and/or 50\% reduction in the total daily dose of insulin secretagogs (that is, sulfonylurea and metiglinides) for patients with baseline HbAlc $\leq 7 \%$.

Statistical analysis. Patient disposition is reported, including all patients who signed the informed consent. All primary and secondary efficacy endpoints were evaluated using the modified intention-to-treat dataset, which included all randomized patients who received at least one dose of study medication and had at least one evaluable endpoint. Patients with no evaluable follow-up data for a particular outcome (for example, NTproBNP) were excluded from those respective analyses. The safety analysis set included all patients who received at least one dose of study medication, and was used for all safety analyses.

Continuous measures were summarized by mean \pm s.d. or median and IQR, and compared using Student's $t$-test or Wilcoxon rank-sum test, as appropriate. Categorical variables were summarized by frequency and percentage and compared using chi-square or Fisher's exact test, as appropriate.

For the primary endpoint, an analysis of covariance model was used to estimate the effect of dapagliflozin relative to placebo on the 12-week KCCQ-CS, adjusting for baseline value, sex, eGFR, T2D status, AF status and LVEF. Restricted cubic splines were included for continuous variables to accommodate nonlinear effects. Supportive analyses were performed examining the effects of dapagliflozin on the components of the primary endpoint (KCCQ-TS and-PL). Several subgroup analyses were prespecified, including age $(<70, \geq 70$ years), sex (male, female), race, T2D status, BMI ( $<$ median, $\geq$ median), baseline NTproBNP ( $<$ median, $\geq$ median), baseline LVEF ( $\leq 60 \%,>60 \%)$, AF status, baseline KCCQ-OS ( $<$ median, $\geq$ median), baseline eGFR $\left(<60, \geq 60 \mathrm{ml} \mathrm{min}^{-1} 1.73 \mathrm{~m}^{-2}\right)$, baseline loop-diuretic dose (furosemide equivalent mean daily dose $\leq 4 \mathrm{mg},>40 \mathrm{mg}$ ), and NYHA class (II, III-IV).

For secondary endpoints, KCCQ-OS was analyzed in a manner analogous to that of the primary endpoint. The unadjusted proportion of patients achieving $\geq 5$-point improvement in KCCQ-CS and -OS at 12 weeks was calculated for the treatment and placebo groups, and a logistic regression model was used to assess the treatment effect adjusted for baseline value, sex, eGFR, T2D, AF and LVEF. A post hoc supportive analysis also compared the proportions of patients that had deterioration ( $>5$-point worsening) or no change, as well as small/moderate (5- to $<10$-point), moderate/large (10- to $<20$-point) and very large (20-point or greater) improvement, in KCCQ-CS between dapagliflozin- and placebo-treated participants, using a Mantel-Haenszel chi-square test. A generalized linear mixed model was used to estimate the treatment effect on 6- and 12-week NTproBNP and BNP values, adjusting for log baseline NTproBNP (or BNP), sex, eGFR, T2D, AF and LVEF, with patient included as a random effect and gamma distribution and log link function used to account for the skewed nature of NTproBNP (or BNP). $\mathrm{HbAlc}$, weight and systolic blood pressure were analyzed in a manner analogous to that of natriuretic peptides, although normal distribution and identity link function were used. Analyses were tested at a two-sided significance level of 5\%, without adjustment for multiple comparisons.

Safety outcomes were assessed using descriptive statistics only, and no $P$ values were calculated. The same approach was used for the number of HF hospitalizations or urgent HF visits.

SAS v.9.4 was used for all analyses (SAS Institute).

Sample size calculations. For the primary endpoint, a sample size of 145 for each group was estimated to achieve $82 \%$ power with $\alpha=0.05$ to detect a 4.7 -point difference in mean KCCQ-CS between dapagliflozin and placebo groups at 12 weeks. The assumptions for this calculation were derived from the DEFINE-HF trial where the mean difference between dapagliflozin and placebo groups was 4.7 points with s.d. $=13.7\left(\right.$ ref. $\left.{ }^{10}\right)$. Assuming a $10 \%$ loss to follow-up, we arrived at a sample size of $\sim 320$ patients.

Reporting Summary. Further information on research design is available in the Nature Research Reporting Summary linked to this article.

\section{Data availability}

Deidentified participant data will be made available on reasonable request 2 years after the date of publication. Requests should be directed to the corresponding author (mkosiborod@saint-lukes.org). Requestors will be required to sign a data access agreement to ensure the appropriate use of the study data.

\section{References}

36. Green, C. P., Porter, C. B., Bresnahan, D. R. \& Spertus, J. A. Development and evaluation of the Kansas City Cardiomyopathy Questionnaire: a new health status measure for heart failure. J. Am. Coll. Cardiol. 35, 1245-1255 (2000). 
37. Guyatt, G. H. et al. The 6-minute walk: a new measure of exercise capacity in patients with chronic heart failure. Can. Med. Assoc. J. 132, 919-923 (1985).

\section{Acknowledgements}

We thank S. Roche, D. Peterman, K. Waybright, M. Jones, A. Blanton, K. Calvin, D. Cocca-Spofford, R. Farrell, J. Ferree, I. Griffin, D. Horner, A. Howarter, B. Garcia, J Huckleberry, M. Hudson, M. Kauffman, N. S. Kuhn, K. Lepkowska, P. Mathiason, D’A. Mauer, R. Newland, H. K. Parson, B. Penn and the Gordon family, L. Pierchala, M. Ramos, E. Ricketts, D. Roshevsky, E. Temponi, V. Shaffer and B. Vargas for their contributions to the PRESERVED-HF Trial. This study was funded by AstraZeneca (grant no. ESR-16-12141, M.N.K.). The funder of the study had no role in the design and conduct of the study, the collection, management, analysis and interpretation of the data, the preparation, review or approval of the manuscript, and the decision to submit the manuscript for publication. This study was an investigator-initiated trial funded by AstraZeneca and conducted by Saint Luke's Mid America Heart Institute independent of the funding source.

\section{Author contributions}

M.N.K. and M.E.N. had full access to all data and take responsibility for the integrity of the data and accuracy of data analysis. Study concept and design was provided by M.N.K., B.A.B., D.W.K. and S.J.S. Acquisition, analysis or interpretation of data was carried out by all authors. Drafting of the manuscript was performed by M.N.K., B.A.B., D.W.K., S.J.S. and M.E.N. All authors critically revised the manuscript for important intellectual content. Statistical analysis was carried out by F.T. Funding was obtained by M.N.K. Administrative, technical or material support was provided by S.L.W. The study was supervised by M.N.K.

\section{Competing interests}

M.N.K. has received research grants from AstraZeneca and Boehringer Ingelheim and is a consultant and/or advisory board member for Amgen, Applied Therapeutics, AstraZeneca, Bayer, Boehringer Ingelheim, Eli Lilly, Esperion Therapeutics, Janssen, Merck (Diabetes and Cardiovascular), Novo Nordisk, Sanofi and Vifor Pharma. M.E.N. is a consultant to Amgen and Roche Vifor and has received speaking honoraria from Abbott. B.A.B. has grant/research Support from National Institutes of Health (NIH)/ NHLBI, Axon, AstraZeneca, Corvia, Medtronic, GlaxoSmithKline, Mesoblast, Novartis and Tenax Therapeutics and consulting/advisory board with Actelion, Amgen, Aria, Boehringer Ingelheim, Edwards Lifesciences, Eli Lilly, Imbria, Janssen, Merck, Novo Nordisk, NGMBio, ShouTi and VADovations. D.W.K. has received honoraria as a consultant for Bayer, Merck, Medtronic, Relypsa, Merck, Corvia Medical, Boehringer Ingelheim, NovoNordisk, AstraZeneca and Novartis, grant funding from Novartis, Bayer, NovoNordisk and AstraZeneca and has stock ownership in Gilead Sciences. S.J.S has research grants from the National Institutes of Health (nos. R01 HL107577, R01
HL127028, R01 HL140731, R01 HL149423), Actelion, AstraZeneca, Corvia, Novartis and Pfizer and consulting fees from Abbott, Actelion, AstraZeneca, Amgen, Aria CV, Axon Therapies, Bayer, Boehringer Ingelheim, Boston Scientific, Bristol Myers Squibb, Cardiora, CVRx, Cytokinetics, Edwards Lifesciences, Eidos, Eisai, Imara Impulse Dynamics, Intellia, Ionis, Ironwood, Lilly, Merck, MyoKardia, Novartis, Novo Nordisk, Pfizer, Prothena, Regeneron, Rivus, Sanofi, Shifamed, Tenax, Tenaya and United Therapeutics. G.U. is partly supported by research grants from the NIH/NATS (no. UL1 TR002378) from the Clinical and Translational Science Award program, and NIH/NIDDK (no. 1P30DK111024-01) from NIH and National Center for Research Resources and has received unrestricted research support for research studies (to Emory University) from Novo Nordisk, AstraZeneca and Dexcom Inc. K.S. is an advisory board member and consultant for Bayer, Boehringer Ingelheim, Bristol Myers Squibb, Cytokinetics, Janssen, Novartis and NovoNordisk and receives honoraria. S.S.K. receives funding from AHA (no. 19TPA34890060) and NIH (no. 1R01HL159250). S.-P.C. is a consultant for Edwards Life Sciences and has received speaking honoraria from Abbott. M.K.K. is an advisory board member for Bayer, Abiomed and CareDx. E.S.S. receives research support from, and is a consultant and speaker for, NovoNordisk, is a consultant and speaker for Boehringer Ingelheim and is a consultant and speaker for Eli Lilly. G.D.L. acknowledges research funding from NIH (nos. R01-HL 151841, R01-HL131029, R01-HL159514), American Heart Association (no. 15GPSGC24800006) and Amgen, Cytokinetics, Applied Therapeutics, AstraZeneca and Sonivie in relation to projects that are distinct from this work; has received honoraria for advisory boards outside of the current study from Pfizer, Merck, Boehringer Ingelheim, Novartis, American Regent, Cyclerion, Cytokinetics and Amgen; and receives royalties from UpToDate for scientific content authorship related to exercise physiology. An AstraZeneca research grant to M.N.K. provided the funding for the PRESERVED-HF trial. None of the other relationships are relevant to the content of this paper. S.L.W., F.T., Y.K., A.O.M., T.K., S.L., C.H.C., L.C., J.J.R., R.A.G., M.P., S.M.J., B.S.C. and M.F. declare no competing interests.

\section{Additional information}

Extended data is available for this paper at https://doi.org/10.1038/s41591-021-01536-x. Supplementary information The online version contains supplementary material available at https://doi.org/10.1038/s41591-021-01536-x.

Correspondence and requests for materials should be addressed to Mikhail N. Kosiborod

Peer review information Nature Medicine thanks John Cleland, Ryan Tedford and Bright Offorha for their contribution to the peer review of this work. Michael Basson and Jennifer Sargent were the handling editors on this article and managed its editorial process and peer review in collaboration with the rest of the editorial team.

Reprints and permissions information is available at www.nature.com/reprints. 


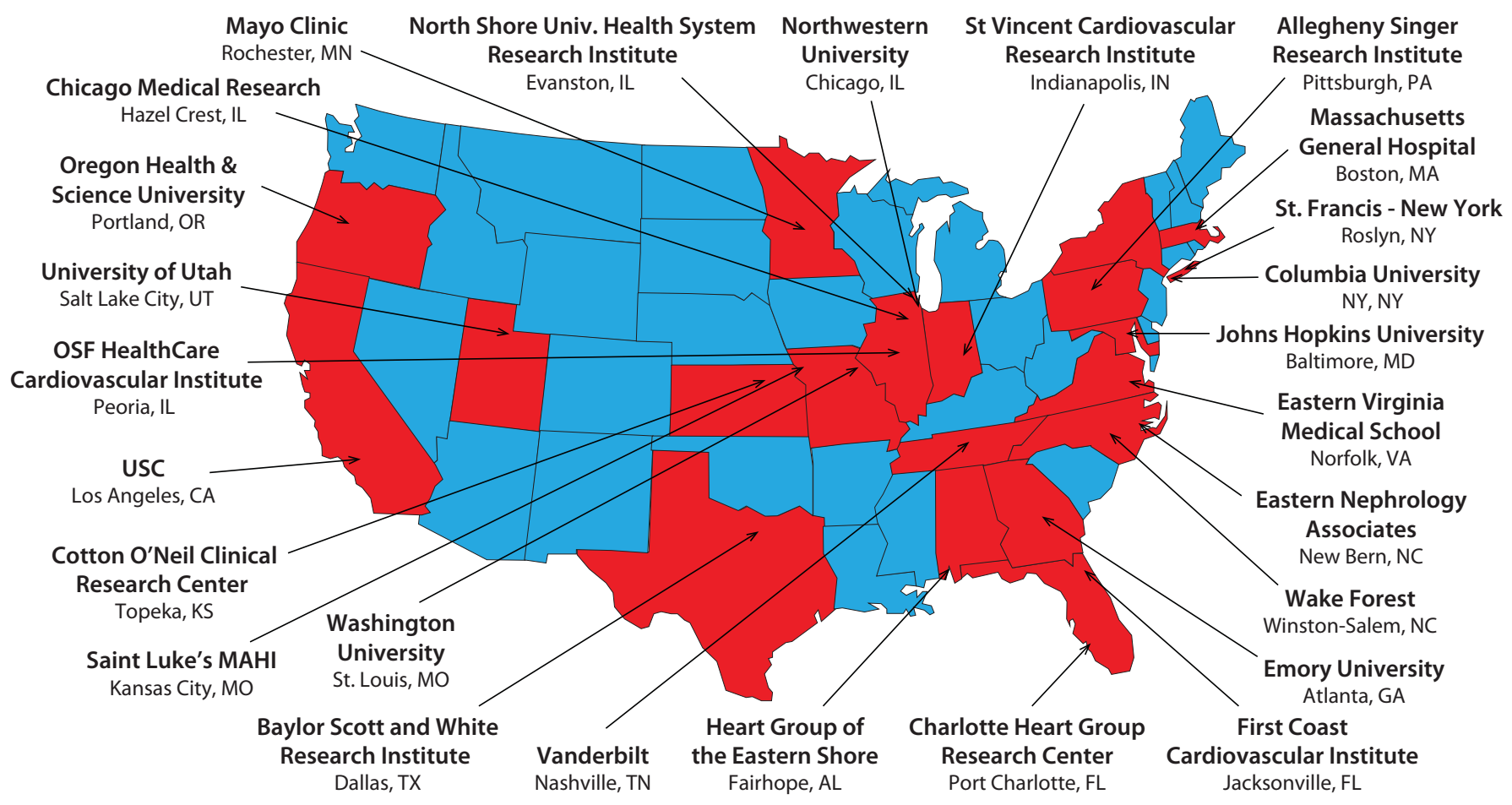

Extended Data Fig. 1 | Map of the study sites. 


\section{ARTICLES}

NATURE MEDICINE

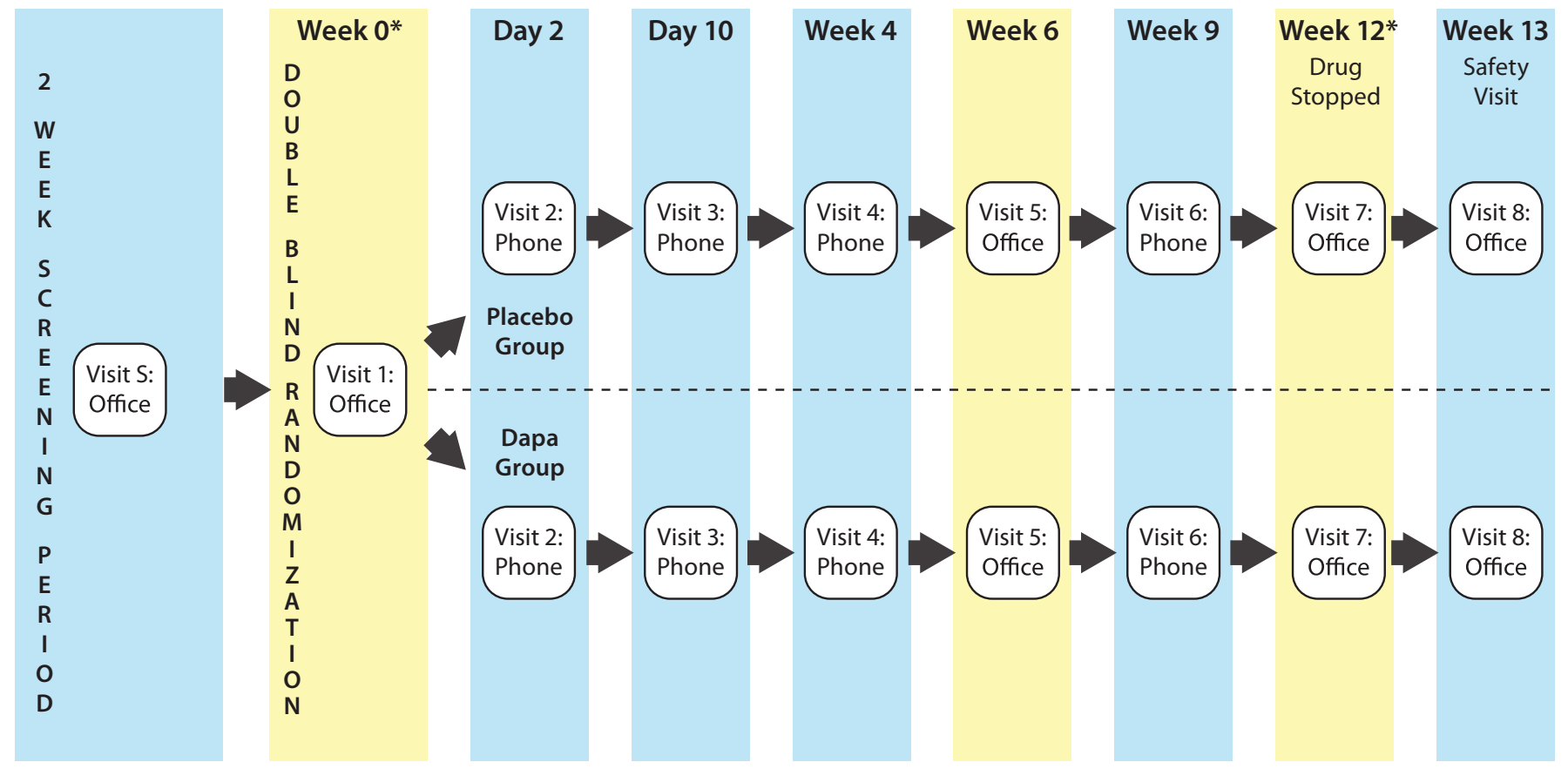

Extended Data Fig. 2 | Trial design and schedule of study visits. 


\section{Reporting Summary}

Nature Research wishes to improve the reproducibility of the work that we publish. This form provides structure for consistency and transparency in reporting. For further information on Nature Research policies, see our Editorial Policies and the Editorial Policy Checklist.

\section{Statistics}

For all statistical analyses, confirm that the following items are present in the figure legend, table legend, main text, or Methods section.

$\mathrm{n} / \mathrm{a}$ Confirmed

\ The exact sample size $(n)$ for each experimental group/condition, given as a discrete number and unit of measurement

$\bigotimes$ A statement on whether measurements were taken from distinct samples or whether the same sample was measured repeatedly

$\triangle$ The statistical test(s) used AND whether they are one- or two-sided

Only common tests should be described solely by name; describe more complex techniques in the Methods section.

$\bigotimes$ A description of all covariates tested

\ A description of any assumptions or corrections, such as tests of normality and adjustment for multiple comparisons

A full description of the statistical parameters including central tendency (e.g. means) or other basic estimates (e.g. regression coefficient) AND variation (e.g. standard deviation) or associated estimates of uncertainty (e.g. confidence intervals)

For null hypothesis testing, the test statistic (e.g. $F, t, r$ ) with confidence intervals, effect sizes, degrees of freedom and $P$ value noted Give $P$ values as exact values whenever suitable.

Х $\square$ For Bayesian analysis, information on the choice of priors and Markov chain Monte Carlo settings

Х $\square$ For hierarchical and complex designs, identification of the appropriate level for tests and full reporting of outcomes

$\square$ Estimates of effect sizes (e.g. Cohen's d, Pearson's $r$ ), indicating how they were calculated

Our web collection on statistics for biologists contains articles on many of the points above.

\section{Software and code}

Policy information about availability of computer code

Data collection REDCap 10.6.1 electronic CRF / ALEA v15.15851.201736 electronic randomization tool

Data analysis SAS 9.4

For manuscripts utilizing custom algorithms or software that are central to the research but not yet described in published literature, software must be made available to editors and

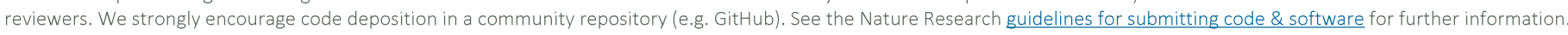

\section{Data}

Policy information about availability of data

All manuscripts must include a data availability statement. This statement should provide the following information, where applicable:

- Accession codes, unique identifiers, or web links for publicly available datasets

- A list of figures that have associated raw data

- A description of any restrictions on data availability

De-identified participant data will be made available on reasonable request two years after the date of publication. Requests should be directed to the corresponding author (mkosiborod@saint-lukes.org). Requestors will be required to sign a data access agreement to ensure the appropriate use of the study data. 
Please select the one below that is the best fit for your research. If you are not sure, read the appropriate sections before making your selection.

\ Life sciences

Behavioural \& social sciences

Ecological, evolutionary \& environmental sciences

For a reference copy of the document with all sections, see nature.com/documents/nr-reporting-summary-flat.pdf

\section{Life sciences study design}

All studies must disclose on these points even when the disclosure is negative.

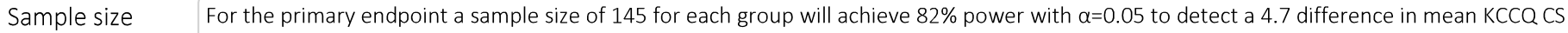
between dapagliflozin group and placebo group at 12 weeks. The assumptions for this calculation was derived from DEFINE-HF trial where the adjusted mean difference between dapagliflozin group and placebo group is 4.7 and the standard deviation is 13.7. Assuming a $10 \%$ loss to follow up, we arrive at a sample size of $\sim 320$ patients.

Data exclusions Patients with no sufficient evaluable data for endpoint ascertainment during follow up were excluded. For example, 20 patients were excluded from the primary endpoint analysis because no followup KCCQ scores were available. This exclusion criterion was pre-specified in the Analysis Data Sets section of the SAP.

Replication Independent validation was performed successfully by a different statistician for the primary and secondary analyses.

Randomization Sharp Clinical Services' web-based IRT (ALEA v15.15851.201736 electronic randomization tool) was utilized to randomize participants to their double-blind treatment allocation. The randomization list was generated and maintained by Sharp Clinical Services and had a fixed block size of 8 stratification groups, based on three stratification questions (diabetes status, atrial fibrillation status fib and participation in the echo substudy). The randomization allocation sequence was implemented through sequentially numbered containers. Sites accessed the IRT through the web at the randomization visit and participants were assigned specific containers by the IRT.

Blinding All the trial team investigators were blinded to treatment allocation throughout the study and during the data analysis. Participants remained blinded to treatment allocation throughout the duration of the study.

\section{Reporting for specific materials, systems and methods}

We require information from authors about some types of materials, experimental systems and methods used in many studies. Here, indicate whether each material, system or method listed is relevant to your study. If you are not sure if a list item applies to your research, read the appropriate section before selecting a response.

\begin{tabular}{l|l} 
Materials \& experimental syste \\
\hline n/a & Involved in the study \\
\hline & $\square$ Antibodies \\
$\square$ & $\square$ Eukaryotic cell lines \\
$\square$ & $\square$ Palaeontology and archaeology \\
\hline & $\square$ Human research participants \\
$\square$ & $\square$ Clinical data \\
$\square$ Dual use research of concern
\end{tabular}

\begin{tabular}{|c|c|}
\hline $\mathrm{n} / \mathrm{a}$ & Involved in the study \\
\hline$\triangle$ & $\square$ ChIP-seq \\
\hline Х & $\square$ Flow cytometry \\
\hline$\bigotimes$ & $\square$ MRI-based neuroimaging \\
\hline
\end{tabular}

Human research participants

Policy information about studies involving human research participants

Population characteristics

Overall, median age was $70.0(63.0,77.0)$ years, $57 \%$ of patients were women, and $30 \%$ African American. The median duration of $\mathrm{HF}$ was $3.0(1.0,6.5)$ years and $56 \%$ had been hospitalized for $\mathrm{HF}$ at least once prior to study enrolment. Overall, $56 \%$ had T2D, and 53\% had atrial fibrillation; median body mass index was 34.7 (interquartile range (IQR), 30.1-41.5). NYHA class II symptoms were present in $57 \%$, with class III-IV symptoms in $42 \%$. Baseline pharmacotherapy included mineralocorticoid antagonists (MRA) in $36 \%$, ACE-I, ARB or ARNI in $62 \%$, and loop diuretics in $88 \%$ of patients. Mean estimated glomerular filtration rate (eGFR) was $55(41,69) \mathrm{mL} / \mathrm{min} / 1.73 \mathrm{m2}$, median NT-proBNP $671.0($ IQR 355.0, 1297.0) pg/ $\mathrm{mL}$ and median left ventricular ejection fraction (LVEF) was $60(55,65)$ percent.

Recruitment Participants were recruited across 26 sites in the United States. Patients were identified from outpatient clinics and inpatient wards. All potentially eligible patients were invited to take part, thereby minimizing any potential self-selection bias.

Ethics oversight

Institutional review boards approved the study at all sites. Participating sites are listed in Supplementary Table 3 and Extended Figure 1. 


\section{Clinical data}

Policy information about clinical studies

All manuscripts should comply with the ICMJE guidelines for publication of clinical research and a completed CONSORT checklist must be included with all submissions

Clinical trial registration This trial is registered with ClinicalTrials.gov, The PRESERVED-HF Trial, NCT 03030235

Study protocol

The study protocol is provided with the manuscript (Supplemental Note).

Data collection

Participants were recruited across 26 sites in the United States (listed in Supplementary Table 3 and Extended Figure 1) over an enrollment period of approximately 50 months. Patients were identified from outpatient clinics and inpatient wards.

Outcomes

All of the primary and secondary outcomes were prespecified in the Statistical Analysis Plan. All statistical analyses performed for all outcome measures were thus predefined. 